\title{
Influence of nuclear reaction rates on the nucleosynthesis in neutrino-driven winds
}

\author{
Carla Fröhlich ${ }^{1, a}$ and Daniel Hatcher ${ }^{2,1}$ \\ ${ }^{1}$ Department of Physics, North Carolina State University, Raleigh, NC 27695-8202, USA \\ ${ }^{2}$ Department of Physics and Astronomy, Appalachian State University, Boone, NC 28608, USA
}

\begin{abstract}
We study the sensitivity of the $v$ p-process abundance pattern to $(\mathrm{n}, \mathrm{p}),(\mathrm{p}, \gamma)$, and $(\mathrm{n}, \gamma)$ rates for nuclei between Ni and Sn. We illustrate our findings for two different initial electron fractions and a representative trajectory. We discuss how these rates influence the abundance pattern and the nuclear flow.
\end{abstract}

\section{Introduction}

Core-collapse supernovae are important contributors to the chemical enrichment of galaxies. They synthesize heavy elements and eject these newly synthesized elements (iron and heavier elements) into the interstellar medium together with elements produced during the star's lifetime. After the explosion, the nascent, hot neutron star cools by emitting neutrinos. These neutrinos interact with the stellar matter and deposit energy, resulting in a supersonic outflow, the neutrino-driven wind. The conditions in the winds are not suitable for the synthesis of the heaviest elements by the r-process. However, lighter heavy elements, such as $\mathrm{Sr}, \mathrm{Y}, \mathrm{Zr}$, can be formed.

Recent hydrodynamic models suggest that very proton-rich conditions are found in the innermost ejected layers of a core-collapse supernova [1-7], due to the interaction of electron neutrinos $v_{\mathrm{e}}$ and anti-neutrinos $\bar{v}_{\mathrm{e}}$ with the ejected, hot, and dense matter at early times. It has been shown that a type of rapid proton-capture process, called the $v$ p-process, unfolds during the cooling phase of such proton-rich ejecta [8-11].

The proton-rich matter cools while being ejected in the core-collapse supernova event and goes from a full nuclear statistical equilibrium (NSE) to two quasi-statistical equilibrium (QSE) groups around ${ }^{56} \mathrm{Ni}$ and $\alpha$-particles. $\alpha$-particles are abundant because the density is comparatively low and hence the $3 \alpha$-rate is too slow to efficiently convert $\alpha$-particles to ${ }^{12} \mathrm{C}$, from where they would be incorporated into the QSE around ${ }^{56} \mathrm{Ni}$. Proton-capture reactions ensue once the temperature drops below QSEtemperatures. This results in sequences of rapid protoncapture reactions competing with $(\gamma, \mathrm{p})$ reactions and $\beta$ decays within an isotonic chain. This is similar to an rpprocess in proton-rich thermonuclear burning on the surface of a mass-accreting neutron star $[12,13]$. However, there are some crucial differences. As the matter is moving

\footnotetext{
a e-mail: cfrohli@ncsu.edu
}

outward in a neutrino wind, the large proton density allows for a small number of neutrons to be produced by $\bar{v}_{\mathrm{e}}$ captures on protons. This permits neutron-induced reactions - such as $(\mathrm{n}, \gamma)$ and $(\mathrm{n}, \mathrm{p})$ - to compete with proton captures, electron captures, and $\beta^{+}$-decays. Especially (n,p) reactions on proton-rich nuclei have cross sections $10-12$ orders of magnitude larger than proton captures. Therefore, already a tiny neutron abundance can cause neutroninduced reaction flows competing with proton captures. The $(\mathrm{n}, \mathrm{p})$ reactions in the $v \mathrm{p}$-process overcome the waiting points in the rp-process which are characterized by slow proton capture rates and long $\beta$-decay (electron capture) lifetimes. Depending on the neutron density $\rho_{\mathrm{n}}$, the $(n, p)$ reactions regulate the flow from one isotonic chain to the next higher as they are faster than the $\beta^{+}$-decays or electron captures. The path of the $v$ p-process lies initially close to the $N=Z$ line and moves towards stability at higher masses.

The importance of the $v$ p-process lies in the fact that it contributes to the abundances of elements above $\mathrm{Ni}$, e.g., to $\mathrm{Sr}, \mathrm{Y}, \mathrm{Zr}$ which have been found to be enhanced in metal-poor stars (e.g., in [14]). Thus, the $v$ p-process may serve as the Light Element Primary Process (LEPP) proposed to explain those abundances [15].

How far in mass the $v$ p-process path reaches depends sensitively on the explosion mechanism, the neutrino emission from the proto-neutron star, and the hydrodynamics governing the ejecta motion, see e.g. [7,16]. On the other hand, many nuclear physics uncertainties are also involved, which additionally affect the isotopic abundance contributions, see e.g. [17]. The reaction path partly lies in a region where nuclear masses are unknown [7, 18] which affects the proton-capture $Q$-values. Furthermore, all involved reaction rates are based on theoretical predictions. Since there is not much experimental information (such as level schemes, deformations, decay schemes, scattering and reaction cross sections) available far from stability, the uncertainties in the rates have to be considered in 
astrophysical studies of the $v$ p-process. A possibility to investigate properties of, and perhaps reactions with, highly unstable nuclei is offered by radioactive ion beam facilities. For the planning of experiments it is useful to have a list of possibly interesting nuclei and reactions for the vp-process.

In this paper, we study (n,p), (p, $\gamma)$, and $(\mathrm{n}, \gamma)$ reactions in $v$ p-process nucleosynthesis. In section 2 , we describe the $v$ p-process model, the nuclear reaction network used, and the reaction rate inputs for the network. The results from systematic variations of individual reaction rates are presented in section 3 . We conclude the article with a discussion in section 4 .

\section{Model and Inputs}

In this study, the $v$ p-process yields are obtained using a post-processing approach of a representative supernova trajectory. The trajectory is from the explosion of a $15 \mathrm{M}_{\odot}$ star [1] and is the same as used in several other $v$ p-process studies $[10,18,19]$. The composition of the ejecta from this trajectory is calculated using a full nuclear reaction network [8], in which we include isotopes up to ${ }^{164} \mathrm{Dy}$. The reaction rates are the same as in [18]. They include experimental rates where available and predictions otherwise. The theoretical n-, p-, and $\alpha$-capture reactions are obtained from the NON-SMOKER code using the masses from the Atomic Mass Evaluation (AME2003) [20], supplemented with mass measurements from [21] and [18]. The theoretical weak interaction rates are taken from [22] and [23]. For the $\beta$-decays, the experimental decay rates from $\mathrm{NuDat}[24]$ are supplemented with the theoretical rates by [25]. Neutrino and antineutrino capture reactions on free nucleons are also taken into account.

We start the nucleosynthesis calculations when the temperature drops below $10 \mathrm{GK}$. The abundances of all isotopes are followed until nuclear reactions freeze-out and matter decays back to stability. The initial abundances are determined from nuclear statistical equilibrium for a fixed electron fraction of $Y_{e}=0.5$ ("standard") or $Y_{e}=0.56$ ("strong"). We assume constant luminosities for neutrinos and antineutrinos. For the antineutrinos we assume a Fermi-Dirac spectrum with a temperature consistent with the hydrodynamical simulation. The neutrino temperature is determined to be consistent with the antineutrino temperature and the chosen initial electron fraction. The subsequent evolution of the electron fraction is performed with the nuclear reaction network that includes electron/positron capture reactions as well as neutrino/antineutrino capture reactions.

\section{Results}

We study the effect of variations in individual nuclear reaction rates on the final $v$ p-process abundances. The important reaction types for the $v \mathrm{p}$-process are (n,p), (p, $\gamma)$, and $(\mathrm{n}, \gamma)$ reactions. The $(\mathrm{p}, \gamma)$ reactions together with the $(\mathrm{n}, \mathrm{p})$ reactions allow for the nuclear flow to proceed to heavier nuclei. The (n,p) reactions take the place of the $\beta^{+}$decays in the rp-process and are of particular importance on even-even $N=Z$ nuclei with long $\beta^{+}$-decay half-lives and small proton-capture cross sections. If the conditions during the expansion result in a low abundance of seed nuclei, neutron-capture reactions become the fastest reactions at temperatures $T<1.5 \mathrm{GK}$ [16]. Toward the end of the evolution, $(\mathrm{n}, \gamma)$ reactions are the fastest reactions, even faster than $\beta^{+}$-decays, and matter moves towards stability along constant proton number $Z$ rather than along constant mass number $A$.

The $v p$-process synthesizes nuclei with $64 \lesssim A \lesssim 110$ on the neutron-deficient side of the valley of $\beta$-stability. It has previously been shown that the nucleus ${ }^{56} \mathrm{Ni}$ acts as seed nucleus for the $v$ p-process and that the final abundances of nuclei with $A>64$ strongly depend on the rate of ${ }^{56} \mathrm{Ni}(\mathrm{n}, \mathrm{p}){ }^{56} \mathrm{Co}[7,17]$. For these reasons, we consider in this study all the nuclei from $\mathrm{Ni}$ to $\mathrm{Sn}$ and from the $N=Z$ line to the most neutron-deficient stable isotope of each element. We investigate individually the (n,p), the (p, $\gamma)$, and the (n, $\gamma)$ reaction on each of these nuclei and determine the change in the final abundances due to a variation in the reaction rate. This is done separately for each of our models ("standard" and "strong").

We perform a baseline calculation for both of our models ("standard" and "strong") using the reaction rates as described in section 2. Figure 1 shows the final abundances for both baseline cases. We denote the final abundance pattern of these calculations with $Y_{\text {baseline }}^{\text {label }}(A)$ where $A$ is the mass number and label $\in$ \{strong, standard $\}$. All the other $v$ p-process calculations use the exact same reaction rate inputs but for the $(n, p),(p, \gamma)$, or $(n, \gamma)$ reaction on a single nucleus. The rate for this reaction and for its reverse reaction are changed by a factor $k=10$ or $k=0.1$. The resulting abundance pattern will be denoted by $Y_{k, x}^{\text {label }}(A)$ where $x$ denotes the reaction type, i.e. $x \in\{(n, p),(p, \gamma),(n, \gamma)\}$. To quantify the difference in the final abundance pattern between a baseline calculation and a calculation with a single reaction changed by a factor $k$, we use the quantity $F$, similar to [26],

$$
F_{k, x}^{\mathrm{label}}=100 \sum_{A} \frac{\left|Y_{k, x}^{\mathrm{label}}(A)-Y_{\text {baseline }}^{\mathrm{label}}(A)\right|}{Y_{\text {baseline }}^{\text {label }}(A)} .
$$

A larger $F$ value represents more deviation in the final abundance pattern from the baseline calculation. A value of $F=0$ would mean that the abundance pattern of the baseline calculation and of the calculation with one changed rate are identical, i.e. the rate change had no effect on the final abundance pattern.

\section{$3.1(n, p)$ reactions}

Figure 2 summarizes the impact of individual (n,p) reaction rates on the $v$ p-process abundance pattern for the two models ("standard" and "strong"). As expected from previous work [7, 17], the (n,p) reactions on ${ }^{56} \mathrm{Ni}$ and ${ }^{64} \mathrm{Ge}$ have a strong impact on the final abundances $(F>1000)$. There are several other (n,p) reactions on even-even $N=Z$ nuclei which also have a significant impact on the final abundance pattern: ${ }^{60} \mathrm{Zn},{ }^{68} \mathrm{Se},{ }^{72} \mathrm{Kr}$, and to a lesser degree also ${ }^{76} \mathrm{Sr}$ and ${ }^{80} \mathrm{Zr}$. In the "strong" case, the (n,p) 


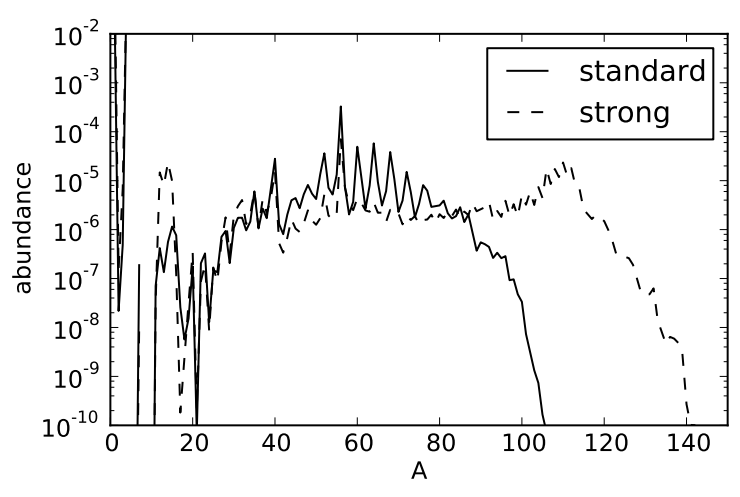

Figure 1. Final abundances versus mass number for the two models considered ("standard" and "strong"). Note that nuclei with mass number $A>110$ are only significantly produced in model "strong".

rates on ${ }^{72} \mathrm{Kr}$, ${ }^{76} \mathrm{Sr}$, and ${ }^{80} \mathrm{Zr}$ are more important than in the "standard" case due to the larger nuclear flow through these nuclei in the "strong" case. The (n,p) rates on the $N+1$ neighbors of the $N=Z$ nuclei are also important. The nuclear flow reaches these nuclei via a $(\mathrm{p}, \gamma)$ reaction immediately after the (n,p) reaction on the $N=Z$ nucleus. For nuclei with $Z>42$ the $v$ p-process path deviates from the $N=Z$ line towards stability. This is reflected in the location of the $(n, p)$ rates with an impact, albeit smaller, on the abundance pattern. We also note a general trend that $(n, p)$ rates on heavier nuclei have a smaller impact on the abundance pattern than those on the nuclei closer to $\mathrm{Ni}$. The nuclear flow reaches the heavier nuclei later when the temperature is already lower and hence the cumulative effect of these rates on the abundance pattern is smaller than for rates on nuclei such as $\mathrm{Ni}, \mathrm{Zn}$, or Ge. The effect of the $(n, p)$ rates on the final abundances is stronger if the rates are decreased than if the rates are increased. The rates for $(n, p)$ reactions on nuclei such as $\mathrm{Ni}$ or $\mathrm{Ge}$ are large and these nuclei are synthesized early during the $v$ p-process. Hence, these nuclei act both as seed nuclei for the $v$ p-process but also as neutron sink. By decreasing the $(n, p)$ rate, the effectiveness of the neutron-sink is decreased and the neutron will be captured by less dominant neutron-deficient nuclei along the $v$ p-process path.

\section{$3.2(p, \gamma)$ and $(n, \gamma)$ reactions}

Generally, the $(\mathrm{p}, \gamma)$ rates do not have a significant impact on the final abundance pattern, both for increases and for decreases in the reaction rates. This is due to the $(\mathrm{p}, \gamma)$ $(\gamma, \mathrm{p})$ equilibrium within isotonic chains during most of the nucleosynthesis. Departures from this equilibrium occur only at late time in the freeze-out.

The $(\mathrm{n}, \gamma)$ rates are important for the $v \mathrm{p}$-process path in two cases: At late times when matter can move towards stability via $(\mathrm{n}, \gamma)$ reactions as previously discussed, or when the nuclear flow reaches $\operatorname{Sn}(Z=50)$ before the freeze-out of the $v$ p-process nucleosynthesis, as seen in our "strong" model. Proton-capture reactions on $\mathrm{Sn}$ iso- topes are unfavorable due to the smaller capture cross sections at the closed shell. Hence, the timescales for radiative neutron-capture reactions becomes shorter than those of any other reaction type. In Figure 4, we can clearly see the importance of neutron-capture reactions on Sn isotopes in our "strong" model. It is also interesting to note that this effect is stronger if the $(n, \gamma)$ rates are increased (rather than decreased), allowing for more neutron-captures to take place at late times when charged-particle reactions are ceasing.

\section{Conclusions}

We have demonstrated the importance of individual $(\mathrm{n}, \mathrm{p})$ reactions, as well $(\mathrm{n}, \gamma)$ reactions on the $v$ p-process abundance pattern. We have shown for two select cases ("standard" and "strong") which reactions have the largest impact on the final abundances. A detailed investigation of the important rates depending on the conditions such as the intitial electron fraction is in preparation. This information combined with information on the relevant energy and the ground-state contribution to the total rate, can be used to plan future experimental investigations of rate isotopes.

CF acknowledges support from the Department of Energy through an Early Career Award (DOE grant no. SC0010263) and through the Topical Collaboration in Nuclear Science "Neutrinos and Nucleosynthesis in Hot and Dense Matter" (DOE grant no. DE-SC0004786). Partial support comes from the National Science Foundation through a Research Experience for Undergraduates grant (NSF Award AST-1062736).

\section{References}

[1] H.T. Janka, R. Buras, M. Rampp, Nuclear Physics A 718, 269 (2003)

[2] M. Liebendörfer, A. Mezzacappa, O.E.B. Messer, G. Martinez-Pinedo, W.R. Hix, F.K. Thielemann, Nuclear Physics A 719, 144 (2003)

[3] T. Fischer, S.C. Whitehouse, A. Mezzacappa, F.K. Thielemann, M. Liebendörfer, Astron. \& Astrophys. 517, A80 (2010)

[4] L. Hüdepohl, B. Müller, H.T. Janka, A. Marek, G.G. Raffelt, Phys. Rev. Lett. 104, 251101 (2010)

[5] T. Fischer, I. Sagert, G. Pagliara, M. Hempel, J. Schaffner-Bielich, T. Rauscher, F.K. Thielemann, R. Käppeli, G. Martínez-Pinedo, M. Liebendörfer, Astrophys. J. Suppl. 194, 39 (2011)

[6] N. Nishimura, T. Fischer, F.K. Thielemann, C. Fröhlich, M. Hempel, R. Käppeli, G. Martínez-Pinedo, T. Rauscher, I. Sagert, C. Winteler, Astrophys. J. 758, 9 (2012)

[7] S. Wanajo, H.T. Janka, S. Kubono, Astrophys. J. 729, 46 (2011)

[8] C. Fröhlich, P. Hauser, M. Liebendörfer, G. Martínez-Pinedo, F.K. Thielemann, E. Bravo, N.T. Zinner, W.R. Hix, K. Langanke, A. Mezzacappa et al., Astrophys. J. 637, 415 (2006) 

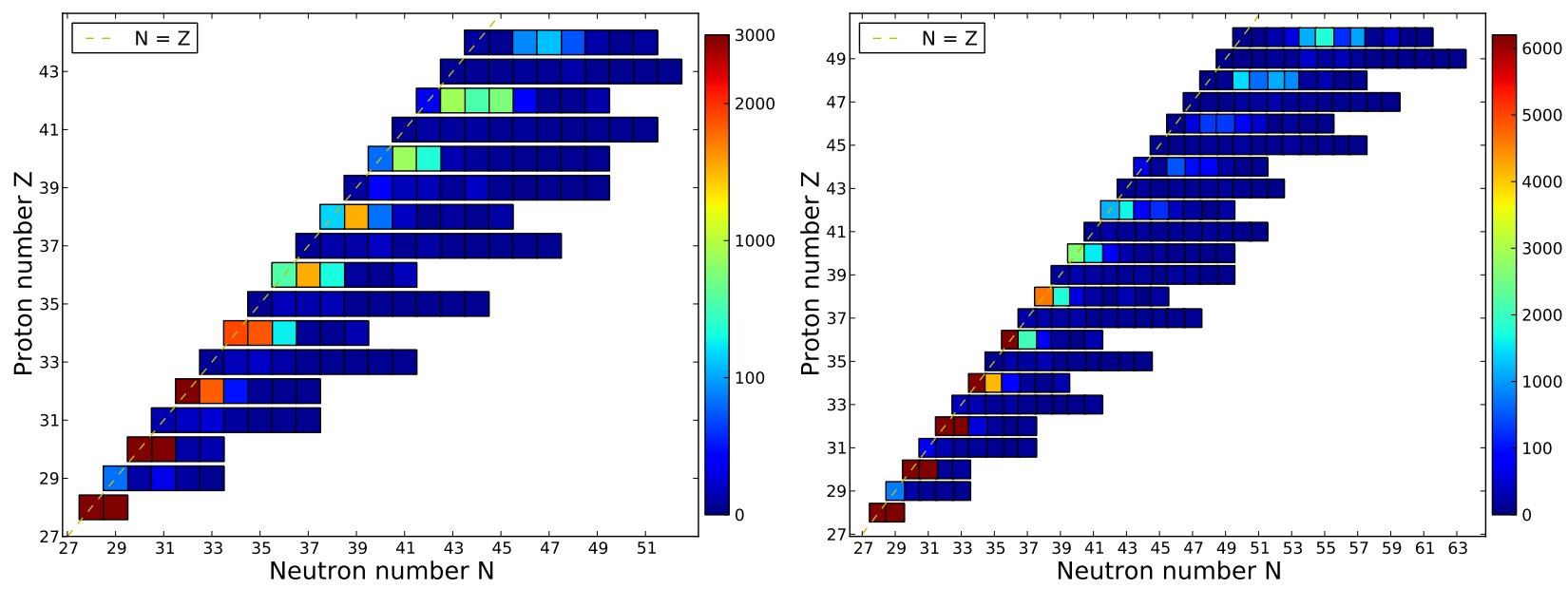

Figure 2. Influence of individual (n,p) reactions on the final abundance pattern for model "standard" (left panel) and "strong" (right panel). The squares indicate isotopes on which we have changed the (n,p) reaction. The color-coding of each square represents the F-value obtained for the calculation with the $(n, p)$ reaction on that particular isotope changed by a factor $k=0.1$. Note the different scale of F-values between the left and the right panels.
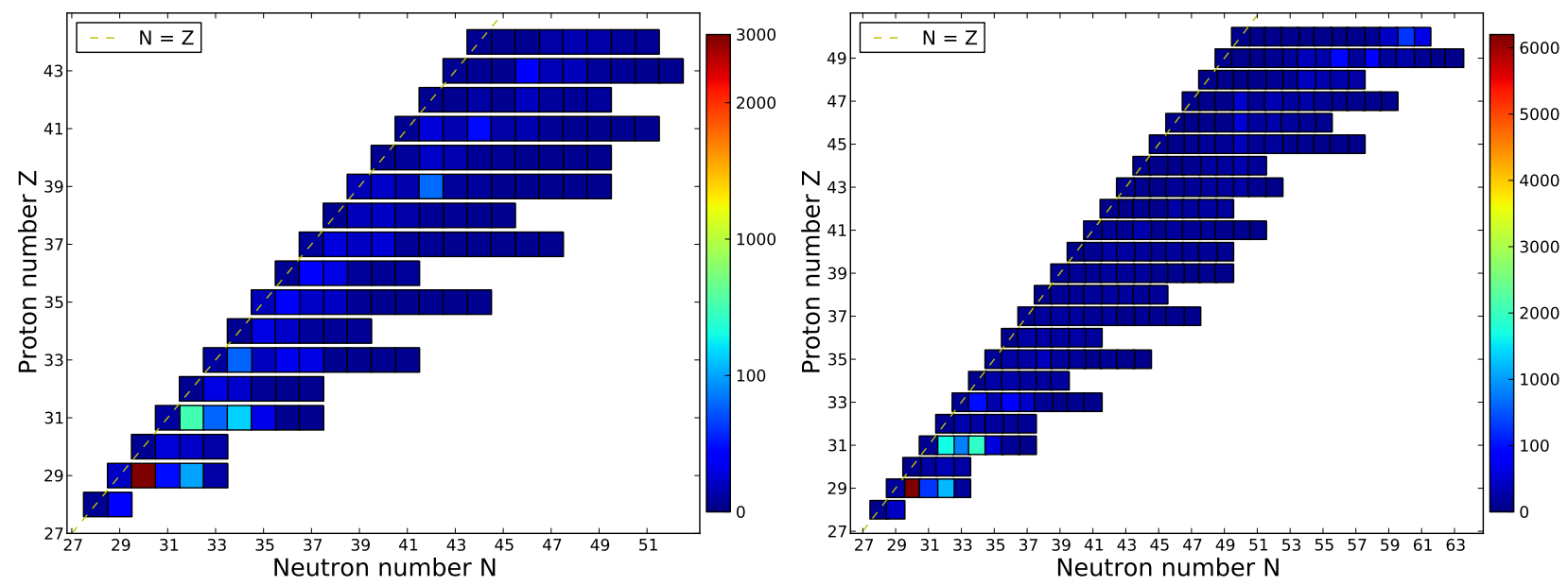

Figure 3. Same as Figure 2 but for $(\mathrm{p}, \gamma)$ reactions.
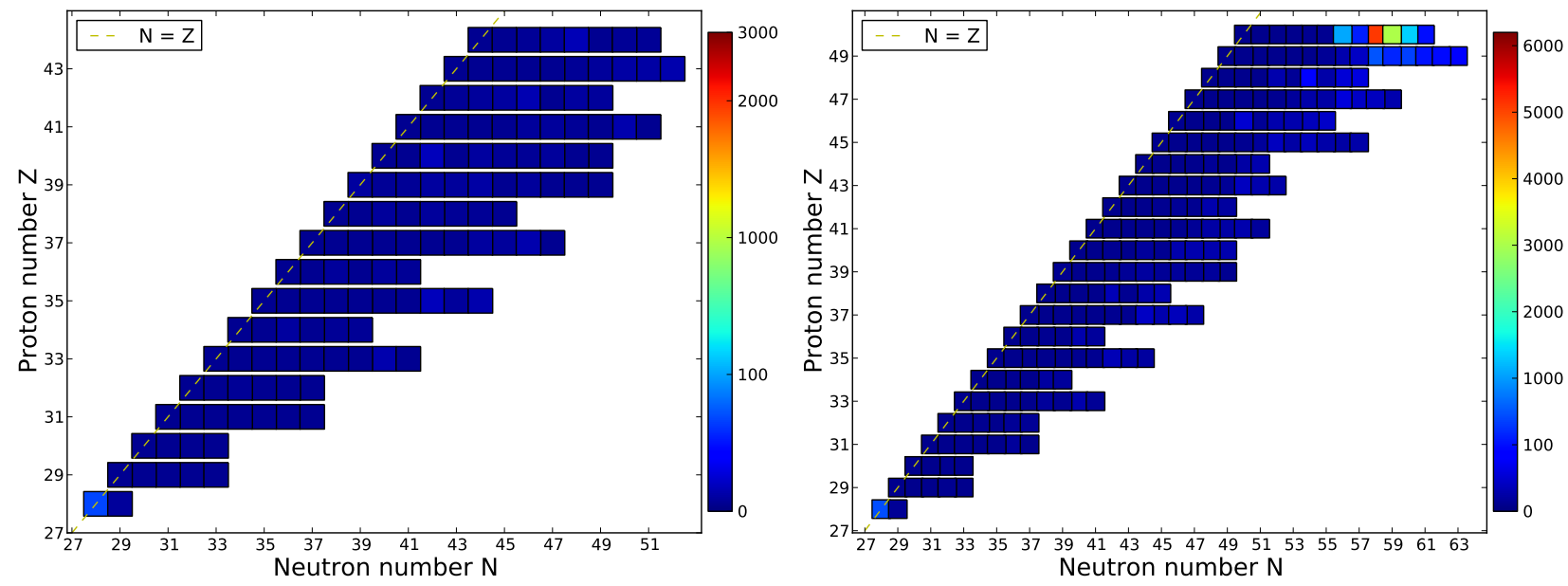

Figure 4. Same as Figure 2 but for (n, $\gamma)$ reactions. 
[9] C. Fröhlich, G. Martínez-Pinedo, M. Liebendörfer, F.K. Thielemann, E. Bravo, W.R. Hix, K. Langanke, N.T. Zinner, Phys. Rev. Lett. 96, 142502 (2006)

[10] J. Pruet, R.D. Hoffman, S.E. Woosley, H.T. Janka, R. Buras, Astrophys. J. 644, 1028 (2006)

[11] S. Wanajo, Astrophys. J. 647, 1323 (2006)

[12] H. Schatz, A. Aprahamian, J. Goerres, M. Wiescher, T. Rauscher, J.F. Rembges, F.K. Thielemann, B. Pfeiffer, P. Moeller, K.L. Kratz et al., Physics Reports 294, 167 (1998)

[13] H. Schatz, A. Aprahamian, V. Barnard, L. Bildsten, A. Cumming, M. Ouellette, T. Rauscher, F.K. Thielemann, M. Wiescher, Physical Review Letters 86, 3471 (2001)

[14] A. Frebel, W. Aoki, N. Christlieb, H. Ando, M. Asplund, P.S. Barklem, T.C. Beers, K. Eriksson, C. Fechner, M.Y. Fujimoto et al., Nature 434, 871 (2005)

[15] C. Travaglio, R. Gallino, E. Arnone, J. Cowan, F. Jordan, C. Sneden, Astrophys. J. 601, 864 (2004)

[16] A. Arcones, C. Fröhlich, G. Martínez-Pinedo, Astrophys. J. 750, 18 (2012)

[17] C. Fröhlich, T. Rauscher, Reaction rate uncertainties and the $v$ p-process, in American Institute of Physics Conference Series, edited by S. Kubono,
T. Hayakawa, T. Kajino, H. Miyatake, T. Motobayashi, K. Nomoto (2012), Vol. 1484 of American Institute of Physics Conference Series, pp. 232-239

[18] C. Weber, V.V. Elomaa, R. Ferrer, C. Fröhlich, D. Ackermann, J. Äystö, G. Audi, L. Batist, K. Blaum, M. Block et al., Physical Review C 78, 054310 (2008)

[19] J.L. Fisker, R.D. Hoffman, J. Pruet, Astrophys. J. Lett. 690, L135 (2009)

[20] G. Audi, A.H. Wapstra, C. Thibault, Nuclear Physics A 729, 337 (2003)

[21] A. Kankainen, L. Batist, S.A. Eliseev, V.V. Elomaa, T. Eronen, U. Hager, J. Hakala, A. Jokinen, I. Moore, Y.N. Novikov et al., European Physical Journal A 29, 271 (2006)

[22] G.M. Fuller, W.A. Fowler, M.J. Newman, Astrophys. J. Suppl. 48, 279 (1982)

[23] K. Langanke, G. Martínez-Pinedo, At. Data. Nucl. Data Tables 79, 1 (2001)

[24] NuDat 2.4, National Nuclear Data Center, http://www.nndc.bnl.gov/nudat2/

[25] P. Möller, B. Pfeiffer, K.L. Kratz, Phys. Rev. C. 67, 055802 (2003)

[26] M.R. Mumpower, G.C. McLaughlin, R. Surman, Phys. Rev. C. 86, 035803 (2012) 
